\title{
Estimativas de repetibilidade de caracteres de produção em laranjeiras-doces no Acre
}

\author{
Jacson Rondinelli da Silva Negreiros ${ }^{(1)}$, Lauro Lessa Saraiva ${ }^{(1)}$, Tadário Kamel de Oliveira(1), \\ Virgínia de Souza Álvares ${ }^{(1)}$ e Givanildo Roncatto(1)
}

(1)Embrapa Acre, BR 364, Km 14, Caixa Postal 321, CEP 69914-220 Rio Branco, AC. E-mail: jacson@cpafac.embrapa.br, laurolessa@yahoo.com.br,
tadario@cpafac.embrapa.br, virginia@cpafac.embrapa.br, givanildo@cpafac.embrapa.br

Resumo - O objetivo deste trabalho foi estimar o coeficiente de repetibilidade em laranjeiras-doces e o número mínimo de avaliações a serem feitas para a determinação do valor real dos indivíduos. De março a junho de 1999, foram coletadas borbulhas de laranjeiras pé-franco, em fase de produção, em nove municípios do Acre. As borbulhas foram enxertadas sobre porta-enxertos de limoeiro 'Cravo', o que resultou em 54 clones. Esses clones foram avaliados em conjunto com a cultivar Aquiri, recomendada para o Estado. O delineamento experimental foi o de blocos ao acaso, com 55 tratamentos (55 clones), três repetições, com uma planta por parcela. O número total de frutos por planta, a produção de frutos por planta e o peso médio de fruto foram avaliados em 2002, 2003, 2004, 2005, 2006 e 2008. Foram realizadas as análises de variância, de componentes principais e estrutural. A estimativa de repetibilidade para peso médio do fruto demonstrou regularidade na classificação dos clones, de um ciclo para outro, e foram necessários cinco ciclos de avaliação para a predição do valor real dos indivíduos, com acurácia de 90\%, pelo método dos componentes principais (matriz de co-variância). Apesar de as características número de frutos total por planta e produção de frutos por planta serem influenciadas pelo ambiente, oito e nove medições, respectivamente, permitem obter coeficientes de determinação de $95 \%$.

Termos para indexação: Citrus sinensis, análise estrutural, análise multivariada, melhoramento genético, seleção clonal.

\section{Estimate of repeatability of traits of sweet orange tree production in Acre, Brazil}

\begin{abstract}
The objective of this study was to estimate the repeatability coefficient in sweet orange and the number of repeated measures that should be performed for an efficient selection of genotypes. From March to June 1999, chips were collected from producing sweet orange seedlings, in nine municipalities of Acre, Brazil, and were grafted on 'Rangpur' lime rootstock, resulting in 54 clones, which were evaluated together with the cultivar Aquiri, recommended for the state. The experiment was carried out in a randomized complete blocks design, with 55 treatments, three replicates and one plant per plot. Fruit number per tree, fruit production per tree and average fruit weight were evaluated in 2002, 2003, 2004, 2005, 2006 and 2008. Analysis of variance, analysis of principal components, and structural analysis were performed. The estimate of repeatability for average fruit weight showed regularity in the ranking of clones, from one cycle to another, and five cycles of assessments were necessary to predict the real value of clones, with accuracy of $90 \%$, when principal components method (matrix of co-variance) was used. Although the number of fruits per plant and fruit yield per plant are influenced by the environment, eight and nine measurements, respectively, would achieve determination coefficients of $95 \%$.
\end{abstract}

Index terms: Citrus sinensis, structural analysis, multivariate analysis, plant breeding, clone selection.

\section{Introdução}

As laranjeiras-doces [Citrus sinensis (L.) Osbeck] compreendem o grupo mais importante de frutas cítricas cultivadas (Roberto et al., 1999). O Brasil é o maior produtor mundial da fruta $(18,06$ milhões de toneladas) e também o maior exportador mundial de suco concentrado de laranja, responsável por $81 \%$ do comércio mundial, com movimentação financeira em torno de US\$ 1 bilhão, em 2005 (Agrianual, 2008).

No Estado do Acre, a laranjeira-doce representa a segunda frutífera de maior expressão comercial, superada somente pela cultura da bananeira (IBGE, 2007). O Estado apresenta boas características edafoclimáticas para a exploração da citricultura; no entanto, a produção não atende à demanda do mercado 
local, que depende da importação, em larga escala, de frutas de outros estados brasileiros, principalmente de São Paulo (Gondim et al., 2001). Aliado ao manejo inadequado da cultura, os principais fatores limitantes à expansão do agronegócio citrícola no Acre, segundo Ledo et al. (1999), seriam a baixa qualidade das mudas, muitas vezes oriundas de outras regiões do País, e a inexistência de informações sobre o comportamento de cultivares nas condições de clima e solo do Estado.

Uma das estratégias para a superação desse desafio é a recomendação de cultivares adaptadas, que sejam produtivas e que apresentem frutos de boa qualidade físico-química. Assim, os programas de melhoramento genético dos citros são dirigidos, basicamente, para a obtenção de cultivares superiores de copa e portaenxerto, e o método mais rápido e eficaz para se alcançar esse objetivo é a introdução de germoplasma, de várias partes do Brasil e de outros países, com posterior seleção de plantas com características desejáveis e que se adaptem aos sistemas de produção utilizados pelos citricultores (Rocha et al., 1992).

Durante o processo de seleção de plantas, com vistas ao lançamento de cultivares ou à escolha de parentais para recombinação, é importante que se tenha certeza da superioridade genética dos indivíduos. Para tanto, são realizadas, muitas vezes, medições repetidas no mesmo indivíduo. É importante, no entanto, o conhecimento do número de medições necessárias, para que sejam selecionados os indivíduos geneticamente superiores, ao menor custo possível (Lopes et al., 2001).

Ao se escolher um genótipo, espera-se que seu desempenho inicial persista durante toda a sua vida. Essa expectativa pode ser verificada pelo coeficiente de repetibilidade de uma característica. A repetibilidade expressa a proporção da variância total, que é explicada pelas variações proporcionadas pelo genótipo e pelas alterações permanentes, atribuídas a um ambiente comum (Abeywardena, 1972; Cruz et al., 2004).

Segundo Vencovsky (1973), o coeficiente de repetibilidade é utilizado em plantas perenes, no estudo de características que se expressam mais de uma vez no decorrer da sua vida. Baseia-se na tomada de mais de uma observação fenotípica de cada indivíduo, sem se utilizarem progênies, com a finalidade de medir a capacidade que eles têm de repetir a expressão do caráter.

No melhoramento de plantas perenes, o estudo de repetibilidade é imprescindível, pois representa o máximo valor que a herdabilidade de um caráter no sentido amplo pode atingir (Falconer, 1987; Cruz et al., 2004), e é usado para determinar o número de observações fenotípicas que devem ser feitas, em cada indivíduo, para que a discriminação ou seleção fenotípica entre genótipos seja realizada eficientemente e com reduzido custo e mão-de-obra. Coeficientes de repetibilidade têm sido estimados em frutíferas como coqueiro (Siqueira, 1982), cupuaçuzeiro (Fonseca et al., 1990; Costa et al., 1997), cacaueiro (Dias \& Kageyama, 1998), cajueiro (Cavalcanti et al., 2000), aceroleira (Lopes et al., 2001; Paiva et al., 2001), goiabeira (Degenhardt et al., 2002) e mangueira (Costa, 2003).

Em laranjeiras-doces, não há trabalhos com análise de medidas repetidas mesmo sendo possível, por meio dessa ferramenta, determinar-se o número mínimo de observações fenotípicas que devem ser feitas em cada indivíduo e aumentar a eficiência da seleção de genótipos com baixo custo.

O objetivo deste trabalho foi estimar os coeficientes de repetibilidade de caracteres produtivos, em laranjeiras-doces, e o número mínimo de avaliações a serem feitas para determinação do valor real dos indivíduos.

\section{Material e Métodos}

O trabalho foi conduzido no Campo Experimental da Embrapa Acre, em Rio Branco, AC, a 10 $11^{\prime} 30^{\prime \prime} \mathrm{S}$, $67^{\circ} 42^{\prime} 18^{\prime \prime} \mathrm{W}$, e altitude de aproximadamente $160 \mathrm{~m}$. $\mathrm{O}$ clima da região é AWI (quente e úmido), segundo Köppen, com temperaturas máxima de $30,92^{\circ} \mathrm{C}$ e mínima de $20,84^{\circ} \mathrm{C}$, precipitação anual de $1.648,94 \mathrm{~mm}$, e umidade relativa de $83 \%$ (Agritempo, 2008). O solo da área experimental foi classificado como Argissolo Vermelho-Amarelo distrófico, de textura média e bem drenado.

No período de março a junho de 1999, realizaramse visitas a propriedades antigas e projetos de assentamento do Instituto Nacional de Colonização e Reforma Agrária, que possuíam pés-francos (plantas oriundas de sementes) de laranjeiras-doces, ainda em fase de produção. Das plantas selecionadas, retiraramse borbulhas (gemas), que foram trazidas ao campo experimental da Embrapa Acre, para a enxertia sobre porta-enxerto de limoeiro 'Cravo' (C. limonia Osbeck). O material foi coletado nos municípios de Plácido de Castro, Senador Guiomard, Capixaba, Xapuri, Sena Madureira, Brasiléia, Epitaciolândia, Porto Acre e Rio 
Branco, no total de 54 clones, além da cultivar Aquiri, recomendada pela Embrapa Acre, cujas mudas foram produzidas simultaneamente com as dos demais clones.

O plantio foi realizado em fevereiro de 2000 , no espaçamento $8 \times 8 \mathrm{~m}$. O delineamento experimental adotado foi o de blocos ao acaso, com 55 tratamentos (55 clones), três repetições, com uma planta útil por parcela, no total de 165 plantas úteis e 56 plantas nas bordaduras. O cultivo foi conduzido sem irrigação, com uso dos tratos culturais recomendados para a cultura dos citros (Petto Neto, 1991).

As características avaliadas foram: número de frutos total por planta (NTF), produção de frutos por planta (PFP) e peso médio de fruto (PMF), em 2002, 2003, 2004, 2005, 2006 e 2008.

As metodologias aplicadas para a estimação do coeficiente de repetibilidade consistiram: na utilização da análise de variância, em que o efeito temporário do ambiente é removido do erro, conforme Cruz et al. (2004); na análise dos componentes principais (CPCOV), obtidos da matriz de correlação e da matriz de variâncias e co-variâncias fenotípicas (Abeywardena, 1972; Rutledge, 1974); e na análise estrutural (AECOR), baseada no autovalor teórico da matriz de correlação ou correlação média (Mansour et al., 1981) (Tabela 1).

O modelo estatístico utilizado para a análise de variância (Cruz et al., 2004) foi: $Y_{i j}=\mu+g_{i}+a_{j}+\varepsilon_{i j}$, em que: $Y_{\mathrm{ij}}$ é o caráter do i-ésimo clone, no j-ésimo ano de medição; $\mu$ é a média geral; $\mathrm{g}_{\mathrm{i}}$ é o efeito aleatório do i-ésimo clone, sob a influência do ambiente permanente ( $\mathrm{i}=1,2, \ldots, 55$ clones); $\mathrm{a}_{\mathrm{j}}$ é o efeito da j-ésima medição $(\mathrm{j}=1,2, \ldots, \eta)$; e $\varepsilon_{\mathrm{ij}}$ é o erro experimental associado ao caráter.

Para cada característica avaliada, o número mínimo de medições necessárias para predizer o valor real dos indivíduos, com base em um coeficiente de determinação $\left(\mathrm{R}^{2}\right)$ pré-estabelecido $(0,85,0,90$ e 0,95$)$, foi calculado por meio da seguinte expressão (Cruz et al., 2004): $\eta_{\mathrm{o}}=\mathrm{R}^{2}(1-\mathrm{r}) /\left(1-\mathrm{R}^{2}\right)$ r, em que: $\eta_{\mathrm{o}}$ é o número de medições para predição do valor real, e $\mathrm{r}$ é o coeficiente de repetibilidade, obtido de acordo com um dos diferentes métodos utilizados.

$\mathrm{O}$ coeficiente de determinação genotípica $\left(\mathrm{R}^{2}\right)$, que representa a percentagem de certeza da predição do valor real, dos indivíduos selecionados com base em $\eta$ medições, foi obtido ela expressão: $\mathrm{R}^{2}=\eta \mathrm{r} /[1+\mathrm{r}(\eta-1)]$, em que $\eta$ é o número de medições e r é o coeficiente de repetibilidade, obtido de acordo com um dos diferentes métodos utilizados.

A análise estatística dos dados foi realizada com o programa computacional GENES, versão 2007 (Cruz, 2001).

\section{Resultados e Discussão}

As estimativas do coeficiente de repetibilidade, obtidas para as três características, por meio das quatro metodologias utilizadas, encontram-se na Tabela 2. Pode-se verificar que o método da análise de variância, para estimação do coeficiente de repetibilidade, é inferior aos demais métodos, para as três características analisadas. Observou-se, ainda, que as estimativas obtidas por meio da CPCOV obtiveram os maiores coeficientes de repetibilidade. Esse resultado implica que as plantas de laranjeira apresentaram, neste trabalho, um comportamento cíclico, ou seja, uma alternância de produção (Spósito et al., 1998; Cruz et al., 2004; Stenzel et al., 2005). Quando o fator periodicidade é significativo, as medidas podem ser afetadas por mudanças de ordem fisiológica,

Tabela 1. Processos de estimação do coeficiente de repetibilidade (r) e seus respectivos estimadores.

\begin{tabular}{lc}
\hline Processo de estimação & Estimador $^{(1)}$ \\
\hline Análise de variância na qual o efeito temporário do ambiente é removido do erro (ANOVA) & $\hat{\sigma}_{\mathrm{g}}^{2} / \hat{\sigma}_{\mathrm{y}}^{2}$ \\
Componentes principais obtidos da matriz de variâncias e covariâncias fenotípicas (CPCOV) & $\frac{\hat{\lambda}_{1}-\hat{\sigma}_{\mathrm{y}}^{2}}{\hat{\sigma}_{\mathrm{y}}^{2}(\eta-1)}$ \\
Componentes principais obtidos da matriz de correlação (CPCOR) & $\hat{\lambda}_{1}^{-1 / \eta-1}$ \\
Análise estrutural com base no autovalor teórico da matriz de correlação ( $\hat{\mathrm{R}})$ ou correlação média (AECOR) & $\left(\hat{\alpha}^{\prime} \hat{\mathrm{R}} \hat{\alpha}-1\right) / \eta-1$ \\
\hline
\end{tabular}

(1) $\hat{\sigma}^{2}=\hat{\sigma}^{2}+\hat{\sigma}_{g}^{2} ; \hat{\sigma}^{2}$ : variância fenotípica; $\sigma^{2}$ : variância ambiental; $\sigma_{g}^{2}$ : variância genotípica; $\hat{\lambda}_{1}$ : autovalor da matriz de covariância $(\hat{\Gamma})$ ou da matriz de correlação $\hat{R}$, associado ao autovetor, cujos elementos têm o mesmo sinal e magnitudes semelhantes; $\hat{\alpha}$ : autovetor associado ao maior autovalor de $\hat{R}$; $\eta$ : número de medições. 
regulares, irregulares ou sistemáticas. Como esse efeito pode variar de maneira e intensidade diferentes entre os genótipos, o método da análise de variância (ANOVA), usualmente utilizado para estimar o coeficiente de repetibilidade, não permite isolar o fator periodicidade que, quando ocorre, fica incluído no erro experimental $\left(\sigma^{2}\right)$, o que eleva seu valor, e causa a subestimação da repetibilidade (Vasconcellos et al., 1985). Nesse caso, o coeficiente de repetibilidade é mais eficientemente estimado pelo método dos componentes principais, que leva em consideração o comportamento cíclico do caráter (Abeywardena, 1972; Kendall, 1975). Por meio dessa metodologia o autovetor, cujos elementos apresentam o mesmo sinal e magnitudes próximas, é aquele que expressa a tendência dos genótipos em manter suas posições relativas nos períodos de medição. $\mathrm{O}$ autovalor que tem este autovetor associado é o estimador do coeficiente de repetibilidade.

Trabalhos em que foram obtidas e comparadas as estimativas de repetibilidade de características de plantas perenes pelo método da ANOVA e por métodos multivariados (análise estrutural e componentes principais), como seringueira (Vasconcellos et al., 1985), pínus (Cornacchia et al., 1995), aceroleira (Lopes et al., 2001) e cajueiro-anão (Cavalcanti et al., 2000), mostraram que as estimativas obtidas pelo método da ANOVA foram sempre inferiores às obtidas pela análise multivariada.

Para o caráter NTF, a estimativa de repetibilidade apresentou variação de 0,267 (ANOVA) a 0,702 (CPCOV), com coeficiente de determinação $\left(\mathrm{R}^{2}\right)$ de 71,803 a $94,284 \%$, respectivamente, o que demonstra certa regularidade na repetição do caráter de uma avaliação para outra. O mesmo foi observado no caráter PFP, com valores de 0,291 (ANOVA) a 0,678 (CPCOV) (Tabela 2).
O caráter peso médio do fruto apresentou alta estimativa para o coeficiente de repetibilidade e determinação (Tabela 2), o que demonstra a acurácia das medições realizadas, a regularidade da superioridade dos indivíduos de um ciclo para outro, e que a expressão dessa característica tem bom controle genético. Esse valor indica que a variância ambiental, para essa característica, foi relativamente baixa, comparada com a variância existente entre plantas. Resultados semelhantes também foram obtidos para essa característica em aceroleira (Lopes et al., 2001), umbuzeiro (Santos, 1999), cupuaçuzeiro (Costa et al., 1997), mangueira (Costa, 2003). Segundo Vencovsky (1973), coeficientes de repetibilidade altos podem ser empregados como parâmetros para medir a capacidade de repetição da expressão do caráter avaliado. Assim, pode-se inferir que o peso médio do fruto pode ser utilizado como parâmetro desejável em métodos simples de melhoramento da laranjeira, a exemplo da seleção fenotípica simples, com boas perspectivas de se obter ganhos genéticos.

Coeficientes de determinação superiores a $90 \%$ foram obtidos para peso médio de fruto, a partir de cinco medições, pelo método dos CPCOV. Para predição de valores reais dos indivíduos, com grau de certeza acima de $95 \%$, são necessárias dez medições pelo mesmo método (Tabela 3 ).

Quanto aos caráteres número de frutos totais por planta e produção de frutos por planta (Tabela 3), para todos os métodos avaliados, foi necessário maior número de medições, em razão das menores estimativas obtidas para $r$, exceto com o método dos componentes principais, obtidos da matriz de variâncias e covariâncias fenotípicas (CPCOV). Por meio desse método, observou-se ser possível, com quatro medições, obter uma precisão de $90 \%$ para ambos os caracteres. Já para o valor de $\mathrm{R}^{2}$ alcançar $95 \%$, seriam necessárias oito e nove medições, para NFT e PFP, respectivamente.

Tabela 2. Estimativas dos coeficientes de repetibilidade $(\hat{\mathrm{r}})$ e seus respectivos coeficientes de determinação $\left(\mathrm{R}^{2}\right)$ do número de frutos total por planta (NTF), produção de frutos por planta (PFP) e peso médio de fruto (PMF), considerando quatro diferentes métodos.

\begin{tabular}{|c|c|c|c|c|c|c|}
\hline \multirow[t]{2}{*}{ Método $^{(1)}$} & \multicolumn{2}{|c|}{ NTF } & \multicolumn{2}{|c|}{ PFP } & \multicolumn{2}{|c|}{ PMF } \\
\hline & $\hat{\mathrm{r}}$ & $\mathrm{R}^{2}(\%)$ & $\hat{\mathrm{r}}$ & $\mathrm{R}^{2}(\%)$ & $\hat{\mathrm{r}}$ & $\mathrm{R}^{2}(\%)$ \\
\hline$\overline{\mathrm{ANOVA}}$ & 0,267 & 71,803 & 0,291 & 74,137 & 0,313 & 76,097 \\
\hline CPCOV & 0,702 & 94,284 & 0,678 & 93,640 & 0,652 & 92,914 \\
\hline CPCOR & 0,356 & 79,430 & 0,350 & 79,054 & 0,601 & 91,322 \\
\hline AECOR & 0,328 & 77,385 & 0,318 & 76,567 & 0,577 & 90,513 \\
\hline
\end{tabular}

(1)ANOVA: análise de variância; CPCOV: componentes principais/variâncias e covariâncias; CPCOR: componentes principais/correlação; AECOR: análise estrutural. 
Tabela 3. Estimativas do número de medições $\left(\eta_{\mathrm{o}}\right)$ necessárias para predição do valor real dos clones quanto aos caracteres número de frutos total por planta (NTF), produção de frutos por planta (PFP) e peso médio de fruto (PMF), considerando quatro diferentes métodos e coeficientes de determinação de 85,90 e $95 \%$.

\begin{tabular}{|c|c|c|c|c|c|c|c|c|c|}
\hline \multirow[t]{2}{*}{ Estimador $^{(1)}$} & \multicolumn{3}{|c|}{ NTF } & \multicolumn{3}{|c|}{ PFP } & \multicolumn{3}{|c|}{ PMF } \\
\hline & $85 \%$ & $90 \%$ & $95 \%$ & $85 \%$ & $90 \%$ & $95 \%$ & $85 \%$ & $90 \%$ & $95 \%$ \\
\hline$\overline{\mathrm{ANOVA}}$ & 15,577 & 24,740 & 52,229 & 13,838 & 21,978 & 46,399 & 12,460 & 19,789 & 41,777 \\
\hline CPCOV & 2,405 & 3,820 & 8,063 & 2,694 & 4,279 & 9,033 & 3,025 & 4,805 & 10,143 \\
\hline CPCOR & 10,273 & 16,315 & 34,443 & 10,510 & 16,692 & 35,239 & 3,769 & 5,986 & 12,638 \\
\hline AECOR & 11,592 & 18,411 & 38,867 & 12,140 & 19,281 & 40,704 & 4,158 & 6,604 & 13,941 \\
\hline
\end{tabular}

(1) ANOVA: análise de variância; CPCOV: componentes principais/variâncias e covariâncias; CPCOR: componentes principais/correlação; AECOR: análise estrutural.

\section{Conclusões}

1. A estimativa do coeficiente de repetibilidade do peso médio do fruto demonstra regularidade na predição da superioridade dos indivíduos de um ciclo para outro, e são necessários cinco ciclos de avaliações para predizer o valor real dos indivíduos, com acurácia de $90 \%$, pelo método dos componentes principais, com base na matriz de variâncias e co-variâncias fenotípicas.

2. Apesar de o número de frutos total por planta e de a produção de frutos por planta sofrerem influência ambiental, oito e nove medições, respectivamente, permitem a obtenção de coeficientes de determinação de $95 \%$.

3. A metodologia da análise de componentes principais, com uso da matriz de variâncias e covariâncias fenotípicas, é mais eficiente para a estimação do coeficiente de repetibilidade das características avaliadas.

\section{Referências}

ABEYWARDENA, V. An application of principal component analysis in genetics. Journal of Genetics, v.61, p.27-51, 1972.

AGRIANUAL. Anuário da agricultura brasileira. São Paulo: Instituto FNP, 2008. 552p.

AGRITEMPO. Dados meteorológicos: Acre. Disponível em: $<$ http://www.agritempo.gov.br/agroclima/sumario $>$. Acesso em: 3 mar. 2008.

CAVALCANTI, J.J.V.; PAIVA, J.R. de; BARROS, L. de M.; CRISÓSTOMO, J.R.; CORRÊA, M.P.F. Repetibilidade de caracteres de produção e porte da planta em clones de cajueiroanão precoce. Pesquisa Agropecuária Brasileira, v.35, p.773-777, 2000.

CORNACCHIA, G.; CRUZ, C.D.; LOBO, P.R.; PIRES, I.E. Estimativas do coeficiente de repetibilidade para características fenotípicas de procedências de Pinus tecunumanii (Schw.) Eguiluz,
Perry e Pinus caribaea var. hondurensis Barret, Golfari. Revista Árvore, v.19, p.333-345, 1995.

COSTA, J.G. da. Estimativas de repetibilidade de alguns caracteres de produção em mangueira. Ciência Rural, v.33, p.263-266, 2003.

COSTA, J.G. da; LEDO, A.S.; OLIVEIRA, M.N. Estimativas de repetibilidade de características de frutos de cupuaçuzeiro no Estado do Acre. Revista Brasileira de Fruticultura, v.19, p.313-318, 1997.

CRUZ, C.D. Programa GENES: versão Windows: aplicativo computacional em genética e estatística. Viçosa: UFV, 2001. $648 \mathrm{p}$.

CRUZ, C.D.; REGAZZI, A.J.; CARNEIRO, P.C.S. Modelos biométricos aplicados ao melhoramento genético. 3.ed. Viçosa: UFV, 2004. v.1. 480p.

DEGENHARDT, J.; DUCROQUET, J.P.; REIS, M.S. dos; GUERRA, M.P.; NODARI, R.O. Efeito de anos e determinação do coeficiente de repetibilidade de características de frutos de goiabeira-serrana. Pesquisa Agropecuária Brasileira, v.37, p.1285-1293, 2002.

DIAS, L.A. dos S.; KAGEYAMA, P.Y. Repeatability and minimum harvest period of cacao (Theobroma cacao L.) in Southern Bahia. Euphytica, v.102, p.29-35, 1998.

FALCONER, D.S. Introdução à genética quantitativa. Viçosa: UFV, 1987. 279p.

FONSECA, C.E.L.; ESCOBAR, J.R.; BUENO, D.M. Variabilidade de alguns caracteres físicos e químicos do fruto do cupuaçuzeiro. Pesquisa Agropecuária Brasileira, v.25, p.1079-1084, 1990.

GONDIM, T.M. de S.; RTZINGER, R.; CUNHA SOBRINHO, A.P. da. Seleção e caracterização de laranjeiras-doces (Citrus sinensis (L.) Osbeck) no Estado do Acre. Revista Brasileira de Fruticultura, v.23, p.451-454, 2001.

IBGE. Sistema IBGE de Recuperação Automática: SIDRA. Disponível em: <http://www.sidra.ibge.gov.br/bda/agric/default. asp? $\mathrm{z}=\mathrm{t} \& \mathrm{o}=11 \& \mathrm{i}=\mathrm{P}>$. Acesso em: 28 nov. 2007.

KENDALL, M.G. Multivariate analysis. New York: MacMillan, 1975. 210p.

LEDO, A. da S.; LEDO, F.J. da S.; RITZINGER, R.; CUNHA SOBRINHO, A.P. da. Porta-enxertos para laranjeiras-doces (Citrus sinensis (L.) Osb.), em Rio Branco, Acre. Pesquisa Agropecuária Brasileira, v.34, p.1211-1216, 1999. 
LOPES, R.; BRUCKNER, C.H.; CRUZ, C.D.; LOPES, M.T.G.; FREITAS, G.B. de. Repetibilidade de características do fruto de aceroleira. Pesquisa Agropecuária Brasileira, v.36, p.507-513, 2001.

MANSOUR, H.; NORDHEIM, E.V.; RUTLEDGE, J.J. Estimators of repeatability. Theoretical and Applied Genetics, v.60, p.151-156, 1981.

PAIVA, J.R. de; RESENDE, M.D.V. de; CORDEIRO, E.R. Avaliação do número de colheitas na produção de progênies de aceroleira, repetibilidade e herdabilidade de caracteres. Revista Brasileira de Fruticultura, v.23, p.102-107, 2001.

PETTO NETO, A. Práticas culturais. In: RODRIGUES, O.; VIÉGAS, F.; POMPEU JUNIOR, J.; AMARO, A.A. (Ed.). Citricultura brasileira. 2.ed. Campinas: Fundação Cargil, 1991. p.476-490.

ROBERTO, S.R.; LIMA, J.E.O. de; CARLOS, E.F. Produtividade inicial da laranjeira 'Valência' (Citrus sinensis (L.) Osbeck) sobre oito porta-enxertos no Estado de São Paulo. Revista Brasileira de Fruticultura, v.21, p.119-122, 1999.

ROCHA, A.F.M.; PASSOS, O.S.; SOARES FILHO, W. dos S.; CUNHA SOBRINHO, A.P. da. Melhoramento genético dos citros: introdução e seleção de cultivares. Cruz das Almas: EmbrapaCNPMF, 1992. 2p. (Embrapa-CNPMF. Citros em Foco, 48).
RUTLEDGE, J.J. Ascaling which removes bias of abeywardena's estimator of repeatability. Journal of Genetics, v.61, p.247-250, 1974.

SANTOS, C.A.F. In situ evaluation of fruit yield and estimation of repeatability coefficient for major fruit traits of umbu tree [Spondias tuberosa (Anacardiaceae)] in the semi-arid region of Brazil. Genetic Resources and Crop Evolution, v.46, p.455-460, 1999.

SIQUEIRA, E.R. Coeficiente da repetibilidade de produção de frutos de coqueiro comum. Pesquisa Agropecuária Brasileira, v.17, p.573-574, 1982.

SPÓSITO, M.B.; CASTRO, P.R.C.; AGUSTÍ, M. Alternância de produção em citros. Laranja, v.19, p.293-304, 1998.

STENZEL, N.M.C.; NEVES, C.S.V.J.; SCHOLZ, M.B. dos S.; GOMES, J.C. Comportamento da laranjeira 'folha murcha' em sete porta-enxertos no noroeste do Paraná. Revista Brasileira de Fruticultura, v.27, p.408-411, 2005.

VASCONCELLOS, M.E.C.; GONÇALVES, P.S.; PAIVA, J.R.; VALOIS, A.C.C. Métodos de estimação do coeficiente de repetibilidade no melhoramento da seringueira. Pesquisa Agropecuária Brasileira, v.20, p.433-437, 1985.

VENCOVSKY, R. Princípios de genética quantitativa. Piracicaba: Esalq, 1973. 97p.

Recebido em 12 de agosto de 2008 e aprovado em 24 de outubro de 2008 